\title{
An UWB-UHF Semi-Passive RFID System for Localization and Tracking Applications
}

\author{
R. D’Errico*, M. Bottazzi ${ }^{\dagger}$, F. Natali ${ }^{\dagger}$, E. Savioli ${ }^{\dagger}$, S. Bartoletti ${ }^{\ddagger}$, A. Conti ${ }^{\ddagger}$, \\ D. Dardari ${ }^{\S}$, N. Decarli ${ }^{\S}$, F. Guidi ${ }^{\S}$, F. Dehmas*, L. Ouvry*, U. Alvarado ฯ,

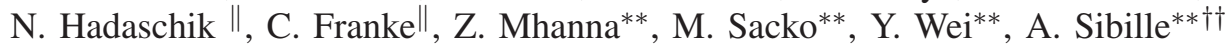 \\ ${ }^{*}$ CEA, LETI, Minatec Campus, 17 rue des Martyrs, 38054 Grenoble, France Email: raffaele.derrico@cea.fr \\ ${ }^{\dagger}$ Datalogic IP Tech, via S.Vitalino 13, 40012 Calderara di Reno (BO), Italy. Email: marco.bottazzi@datalogic.com \\ ${ }_{\ddagger}^{\ddagger}$ ENDIF, CNIT at University of Ferrara. Via Saragat, 144122 Ferrara, Italy. Email: a.conti@iee.org \\ ${ }^{\S}$ CNIT, University of Bologna, via Venezia 52, 47521, Cesena (FC), Italy. Email: ddardari@ieee.org \\ \CEIT, Paseo Manuel Lardizabal 15, 20018 San Sebastian, Spain. Email: ualvarado@ceit.es \\ " Fraunhofer IIS, Nordostpark 93, 90411 Nuremberg, Germany. Email: niels.hadaschik@iis.fraunhofer.de \\ **Telecom ParisTech, 46 rue Barrault 75634 Paris cedex 13, France. Email: alain.sibille@telecom-paristech.fr \\ ${ }^{\dagger}$ ENSTA ParisTech, 32, Boulevard Victor 75015 Paris, France. Email: alain.sibille@ensta.fr
}

\begin{abstract}
We present a novel radio-frequency identification (RFID) system with capability of localization and tracking of passive or semi-passive tags. Localization and tracking features are enabled by backscatter modulation on ultra-wide bandwidth tag's antenna. A ultra-high frequency signal allows the wakeup of the tags enabling the reduction of energy consumption and ensuring compatibility with existing RFID systems. The overall system as well as the reader and tag architectures are introduced. The localization and tracking performance evaluation is presented in some reference scenarios.

Index Terms-Radio Frequency IDentification (RFID), Ultrawide Bandwidth (UWB), Ultra-High Frequency (UHF), antenna backscattering modulation, localization, tracking.
\end{abstract}

\section{INTRODUCTION}

In recent years, a significant number of industrial realities have moved towards the so-called supply chain management (SCM) approach [1], relying on the administration of the various logistics aspects of the company. One of the main requirements of the SCM approach is the visibility of the goods along the chain. More precisely, it is necessary to know what a given object is (the who question), and where it can be found (the where question) at a given instant in time (the when question). Automatic identification (AI) technologies and the existing real time locating systems (RTLS) are not able to provide a complete solution. In fact, both of them suffer from significant limitations. On the one hand AI systems are able to answer to the "who" question, but they are weak on the "where" question. To obtain the complete item visibility, items have to be scanned and a position information must be manually inserted or read from a separate source (e.g., another tag). This aspects severely reduce the efficiency of SCM and introduce uncertainty because of manual operations. On the other hand, RTLS satisfy mainly the location requirement, but fail to manage the identification requirement when the item is out of the RTLS working area. Moreover, the current generation of RTLS are based on active tags. This leads to two deficiencies: a limited battery lifetime and a high cost of the tag. As a consequence, the wide spreading of RTLS systems is still not fully exploited.

Recently it has been shown that impulse radio (IR)-ultrawide bandwidth (UWB) is a very promising technique, which could meet the stringent requirements of passive tag localization in terms of accuracy [2]. In [3] a passive UWB-radiofrequency identification (RFID) scheme has been proposed, showing its potential operating range/data rate trade-off. The advantage of such a technology is to provide the typical accuracy of UWB-RTLS by employing a very simple tag, which adopts backscattering modulation instead of using a complete UWB active transmitter. However one of the most important issues in these systems is the energy supply. Combining UWB (semi-)passive RFID with already existing ultrahigh frequency (UHF) technologies can be a possible solution to exploit energy harvesting [4] or to implement a wakeup in order to increase the battery life of a semi-passive tags. Moreover the UHF module can be employed to ensure compatibility with already existing RFID systems.

In this paper we present an overview on a UHF-UWB semipassive RFID system which enables localization and tracking features, and it is being developed in the scope of the FP7 European Project SELECT [5].

\section{SCENARIO AND REQUIREMENTS}

The objective of the SELECT project are: (a) to develop new-generation UWB-based, semi-passive, low-cost tag compatible with UHF-RFID standard; (b) to design an UWBRTLS based on such tags, capable of offering up to 15 $\mathrm{m}$ of operational range, with sub-meter location accuracy. In particular, the ranging accuracy at a distance of $7.35 \mathrm{~m}$ (considering a constellation of 4 readers placed at $2 \mathrm{~m}$ height, 
in the corners of a $10 \times 10$ squared meters square cell) shall be $12 \mathrm{~cm}$ under the line-of-sight (LOS) condition, corresponding to $20 \mathrm{~cm}$ for $2 \mathrm{D}$ accuracy, within $75 \%$ confidence. These requirements dictate the choice on technologies that have to be used to achieve these goals. Considering the required accuracy, it is necessary to adopt IR-UWB technology, since it is the only technology that offers the necessary precision level [6][9]. Moreover, in order to satisfy the low-cost and low-power consumption requirements, the tag cannot be equipped with a UWB transmitter, as usual in the current generation systems, therefore a backscattering modulation approach is adopted. For the sake of fully satisfying the visibility requirement, the tag has to be improved with standard UHF-RFID capabilities, so that the objects can be tagged with a single SELECT tag in order to be located and tracked when they are inside a SELECT-based facility and to be identified in a conventional UHF-RFID system.

\section{UWB BACKSCATTERING PRINCIPLES}

\section{A. UWB backscattering channel}

The UWB round trip backscattering channel is strongly unfavorable from the energetic point of view, since the received backscattered signal experiences path loss between the reader and the tag twice. Basically, similarly to the radar equation, the distance-dependence of the received signal power scales, in free space, with the 4 th power of the reader-tag distance, which means a detection distance much smaller than for an ordinary communication link. An example of the received signal is shown on Fig. 1, where the plot shows the delay domain backscattering (BS) signal for three states of the load impedance connected to the tag antenna. In one of them

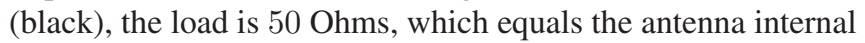
impedance. In this case, the BS signal is called "structural mode" and is dominated by the physical structure of the antenna and its various parts. In the two other cases, we distinctly see that a portion of the signal depends on the load impedance. This part of the BS signal is named "antenna mode" and it is the one that allows differential detection through the tag load impedance state, as detailed in Sec. III-B.

Actually, the round trip BS channel in the delay domain is the result of the convolution between the reader-tag propagation channel convoluted by itself and the BS antenna mode response of the tag. The contours of a complete BS channel model encompassing all these contributions have been described in [10]. Apart from the reader-tag propagation, a very important contributor to the received BS signal is the tag itself. Indeed, it is impossible for a tag to behave as an ideal isotropic scatterer, which would equally receive the reader signal from any direction and would scatter it equally into all directions. The bistatic antenna mode response involves the tag antenna radiation pattern both when it receives and when it retransmits, and unfortunately achieving an isotropic UWB radiator is not possible. Moreover the tag radio-electric behavior is obviously strongly impacted by the presence of the object on which it is attached that may act as a obstruction. Such issues have been investigated in [11], which aimed to construct a



Fig. 1. Example of measured backscattered signal, for three tag load impedance values (free space).

statistical tag performance model, able of accounting for the variability in the characteristics of tags of various designs and for the variability of objects in close interaction. The classical Saleh-Valenzuela wideband channel model, also used in the IEEE 802.15.4a standard [12], is a natural candidate model to describe this contribution and it encompasses several environments, from "gentle" short distance LOS indoor ones to much "harder" ones in industrial environments. Ongoing work targets combining a statistical tag performance model [11] and a multipath propagation model [10], in order to achieve a full BS channel model, able to realistically cover a variety of use cases and propagation environments.

\section{B. UWB passive scheme}

In Fig. 2 we report the architecture proposed in [3] and analyzed in [2], [13] for UWB readers and tags adopting backscatter modulation. This architecture represents the UWB core enabling the backscattering scheme for localization and tracking. In particular, the reader is composed of a transmitter and a receiver section.

During the interrogation cycle, the reader transmits a sequence of UWB pulses modulated by a periodic binary spreading sequence $\left\{d_{n}\right\}$ of period $N_{\mathrm{c}}$ with $d_{n} \in\{-1,1\}$, specific of that particular reader (reader's code). In general, $N_{\mathrm{pc}}$ pulses are associated to each code symbol (chip) of duration $T_{\mathrm{c}}$ seconds. To accommodate the signals backscattered by tags corresponding to an entire packet of $N_{\mathrm{r}}$ bits, the UWB interrogation contains $N_{\mathrm{t}}=N_{\mathrm{r}} N_{\mathrm{s}}$ pulses, where $N_{\mathrm{s}}=N_{\mathrm{c}} N_{\mathrm{pc}}$ is the number of pulses associated to each bit. Pulses are separated by $T_{\mathrm{p}}$ seconds, thus the chip time is $T_{\mathrm{c}}=N_{\mathrm{pc}} T_{\mathrm{p}}$. The pulse repetition period $T_{\mathrm{p}}$ is chosen so that all signals backscattered by the environment are received by the reader before the transmission of the successive pulse (in the SELECT system $\left.T_{\mathrm{p}}=128 \mathrm{~ns}\right)$. Each transmitted pulse is backscattered by the tag's antenna as well as by all the surrounding scatterers present in the environment which form the clutter component.

In Fig. 2, an example of UWB tag architecture employing a 


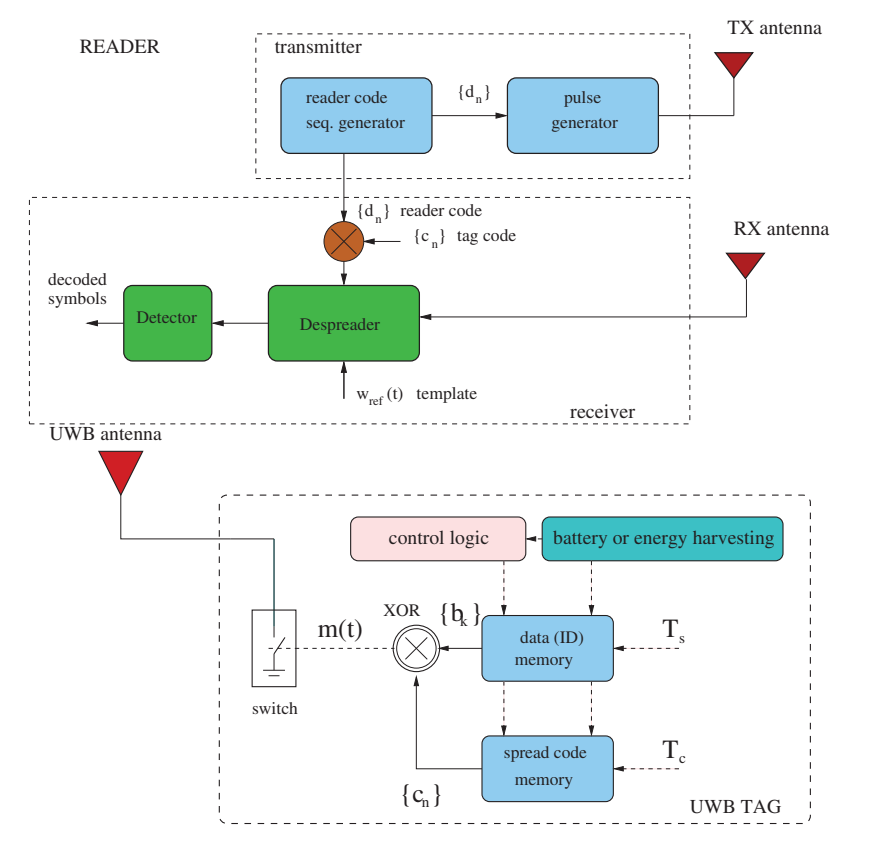

Fig. 2. UWB tag and the reader architectures in backscattering schemes [3].

binary backscatter modulator composed of an UWB switch is shown. The switch is controlled by a micro-controller whose purpose is to change the switch status (short or open circuit) at each chip time $T_{\mathrm{c}}$ according to the data to be transmitted and a zero mean (balanced) periodic tag's code $\left\{c_{n}\right\}$, with $c_{n} \in\{-1,+1\}$, of period $N_{\mathrm{c}}$. The adoption of balanced codes ensures the removal of the clutter [3]. Specifically, each tag information bit $b_{k} \in\{-1,+1\}$ is associated to $N_{\mathrm{s}}$ pulses, thus the symbol time results $T_{\mathrm{s}}=T_{\mathrm{c}} N_{\mathrm{c}}=T_{\mathrm{p}} N_{\mathrm{s}}$. In this way the polarity of the reflected signal changes according to the tag's code during a symbol time, whereas the information symbol (bit) affects the polarity of all pulses composing the sequence each symbol time.

By analyzing the received signal components, it can be noted that the antenna mode scattered component only is modulated by the combination of the tag's and reader's codes $\left\{c_{n}\right\}$ and $\left\{d_{n}\right\}$, whereas all clutter signals components (included the antenna structural mode scattering) are received modulated by the reader's code $\left\{d_{n}\right\}$ only. This property is exploited at the reader receiver section to remove the clutter component through a proper processing, therefore isolating the useful component coming from the intended tag.

An example of possible receiver scheme is that reported in Fig. 2 where a correlator-based demodulator is adopted. In substance this scheme performs a de-spreading operation using the combined code $\left\{c_{n} \otimes d_{n}\right\}$, which identifies both the reader and the desired tag. The performance analysis of the above architecture has been carried out in [13]-[15], where the main design issues related to the presence of clutter, clock synchronization errors, multi-tag and multi-reader interference are addressed.



Fig. 3. SELECT general system architecture.

\section{System ARCHITECTURE}

The overall system architecture is depicted in Fig. 3. It comprises a central unit, readers and tags. Relays are understood herein as devices unconnected to the wired core network: they can be optionally employed in order to provide a coverage enhancement [16]. A UHF reader-tag communication is established to wake-up the UWB tag's antenna load modulator, in order to start backscattering the UWB signal sent by the reader. Optionally a standard UHF-RFID communication can be realized.

\section{A. Reader design}

Readers provide the link between the tags and the backbone hardware allowing for locating the tags via UWB communication. From the hardware perspective, they consist of a motherboard, on which modules are stacked as sketched in Fig. 4. These comprise a UWB transmitter front-end, a UWB receiver front-end and a UHF module. The motherboard mainly consist of a FPGA and a micro-controller: the FPGA handles the massively parallel signal processing, controls the front-ends and communicates with them; the micro-controller enables the internal reader control and communicates with the remote backbone hardware via an Ethernet connection. Furthermore, the ARM Cortex A9 based OMAP platform running LINUX packs the data into LocON [17] protocol packets designed to serve RTLS. The FPGA is a SPARTAN 6 XC6SLX150 from Xilinx that provides the computational force for multiple parallel correlator-banks in order to compute the signal round-trip time (RTT). Additionally, the motherboard provides reference clock and power to all components and has an embedded boot management controller. This controller supports power management and switches the external memory interface (EMIF) to provide the main communication link between OMAP, FPGA, and Flash.

The UWB transmitter module is based on the TCR platform by CEA-LETI [18] and radiates bipolar UWB pulse trains at a rate of $8 \mathrm{MHz}$ according to configurable pulse shapes 


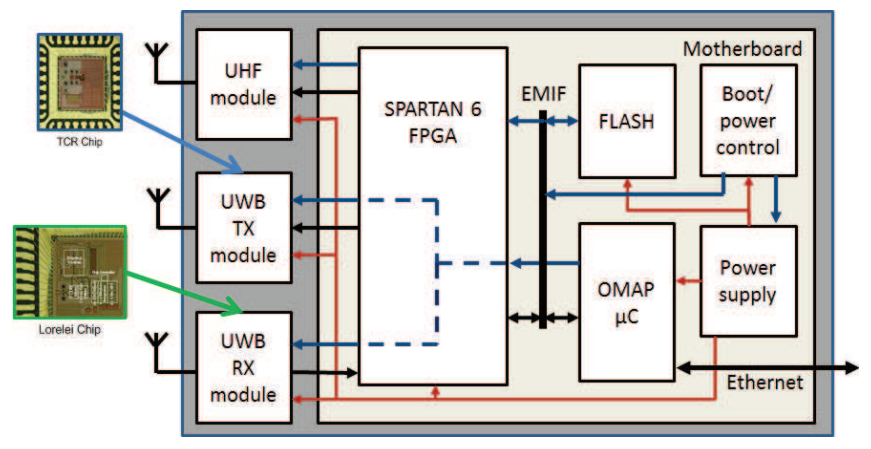

Fig. 4. SELECT reader: black indicates communication, blue configuration links, and red power supply.

and spreading codes. These approximately Gaussian pulses have a $10 \mathrm{~dB}$-bandwidth of $1 \mathrm{GHz}$ and are centered around 4.5 GHz. The UWB receiver, derived from [19], employs double quadrature demodulation together with integration windows of $2 \mathrm{~ns}$. Four fingers with different integration window offsets - tuneable in 1 ns steps - are realized simultaneously allowing for successively scanning the channel response in $1 \mathrm{~ns}$ steps, while sampling at a low-rate of $62.5 \mathrm{MHz}$. In its final version the receiver front-end is equipped with an external 12 bit analog-to-digital converter (ADC) required due to large expected dynamic range of the incoming signal, especially caused by low signal-to-clutter and signal-tointerference ratios. Two identical UWB directive antennas are employed at both transmitter and receiver sides, to obtain a quasi-monostatic set-up. The antennas present an average maximum gain of $7.8 \mathrm{dBi}$ in the band of interest, and an half power beam width (HPBW) of approximately $60^{\circ}$ and $30^{\circ}$ in the E-plane and H-plane, respectively. The use of slight directive antennas allows a link budget enhancement thanks to the antenna gain at receiver side. The antenna gain has to be considered in order to respect the UWB emission masks [20], [21]. A firmware module, as part of the sequential RTT estimation, configures the integration windows of the fingers, and thus, schedules the time bins of interest.

The absence of tag's synchronization would require to search for a large number of tag's code shifts since every tag would be completely asynchronous with respect to the reader's code and the other tag's. As this is computationally infeasible with the available hardware, the readers awake the tags by means of a UHF continuous wave signal sent from the respective module, and the tags start modulating the UWB pulses at the end of the UHF signal. In order to ensure a correct sequence of the operation, hardware modules are synchronized, driven, and configured via the FPGA logic, where the configuration is initialized by the micro-controller.

\section{B. Tag design}

Fig. 5 shows the functional blocks that compose the tag. Two different sections are responsible for signal processing of the UHF and UWB signals. More precisely, the UHF subsystem is composed of a standard EPC Gen2 compliant UHF-RFID IC driven by appropriate control logic, which



Fig. 5. SELECT tag architecture.

makes the tag compatible with standard EPC Gen2 readers. In addition, a passive front-end (Wake-up + SYNC block) detects the presence of a wake-up signal (UHF continuous wave) and generates both the battery activation trigger (wake_up) and the tag synchronization signal $(S Y N C)$. It also comprises an ASK demodulator whose output is used by the control logic to manage false wake-ups and tag shut-down. This way, if a false wake-up is detected, the tag power supply is disabled, putting it back into sleep mode. On the other hand, the power management unit (PM) provides the necessary voltage supplies, references, and currents to optimize the performance and the power consumption of each circuit. It also manages the battery (BATT) usage, by switching the tag on when a wake-up signal is detected, while switching it off if either the message transmission has finished or a false wake-up has been detected.

The UWB section is composed of a control logic unit, a data memory which contains the data bits to be transmitted (tag ID) according to the defined packet structure and the spreading code necessary to allow multiple access and clutter removal. A proper binary signal $m(t)$ drives the RF stage for this purpose. The RF stage consists of a simple UWB switch, which constitutes the backscatter modulator, by switching the UWB antenna load on short or open circuit. The clock is generated by means of a quartz crystal-based oscillator, in order to cope with the stringent frequency (both accuracy and drift) requirements (100 ppm frequency accuracy and $1 \mu \mathrm{s}$ offset).

The UHF and UWB sections are connected to a co-located dual band antenna with two different ports: single-ended for the UWB and differential for the UHF. The normal state of the tag is "sleeping mode" where no current is drawn from the battery and all circuits are off. When a wake-up signal is received through the UHF antenna, the UHF subsystem detects it and generates the wake-up trigger signal. Upon detection, this signal is processed by the power management unit and then the battery is switched-on. From this moment, all voltages and currents are set by the PM unit, and therefore the UWB control logic and memories are powered-up; the clock is also initialized and the UWB switch (i.e., the backscatter modulator) starts operating. 


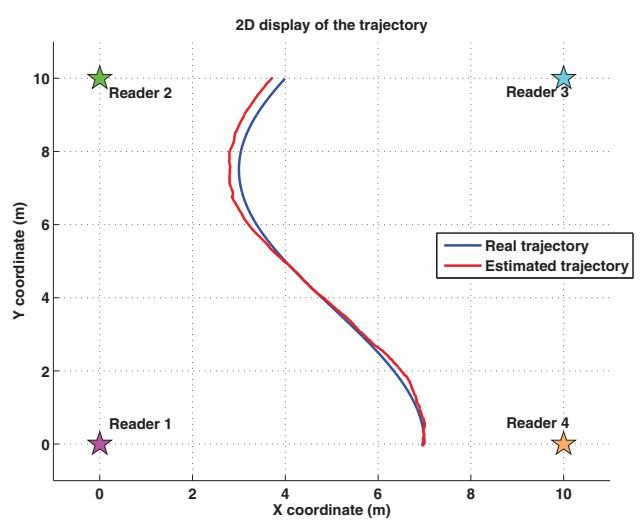

Fig. 6. Example of 2D positioning estimation simulation result [25].

The synchronization signal needed to synchronize all the population of tags under coverage, is derived from the transition of the UHF wake-up signal from "presence" to "absence". During this transition the UHF subsystem generates the synchronization signal (SYNC), which resets the control logic so that the transmission of data bits starts synchronously. The data packet (tag ID) stored in the data memory is transmitted, using the spreading code stored in the spreading code memory. After the communication ends, the control logic ensures the tag's shut down through the power management unit (PM) and the battery is switched-off, putting the tag once again into sleep mode, where no current at all is demanded by any circuit.

\section{Localization Performance Evaluation}

Localization and navigation algorithms rely on time-ofarrival (TOA) estimations of backscattered signals received at each reader in the network [6], [22], [23]. Tracking algorithms for dynamic tags are based on extended Kalman filters (EKFs) and particle filters (PFs). For static tags a least squares (LS) algorithm is considered. The performance is given in terms of localization root mean square error (RMSE), localization error outage (LEO), and navigation error outage (NEO) [24]. The influence of different operating scenarios and of the localization update rate $1 / T_{\mathrm{r}}$, where $T_{\mathrm{r}}$ is the time between two consecutive TOA estimations, is investigated.

A bi-dimensional reference scenario composed of a $10 \times 10$ squared meters room with four readers located at the corners is chosen. We consider a channel model given by a twoway IEEE 802.15.4a channel with backscattering antenna response. Details of signal processing and TOA estimation can be found in [25]. Figure 6 shows an example of real and estimated trajectory obtained by simulation when an EKFbased algorithm is employed. When considering a tag moving with a maximum speed of $1 \mathrm{~m} / \mathrm{s}$, in the absence of interferers, the RMSE over 100 random trajectories is $0.17 \mathrm{~m}$ and $0.39 \mathrm{~m}$ for $T_{\mathrm{r}}=0.5 \mathrm{~s}$ and $T_{\mathrm{r}}=1 \mathrm{~s}$, respectively.

Measurement campaigns have been carried out to characterize backscattering channel and antenna response in different environments (warehouse-like, room-like, and laboratory-like). These experiments have been made under different multipath

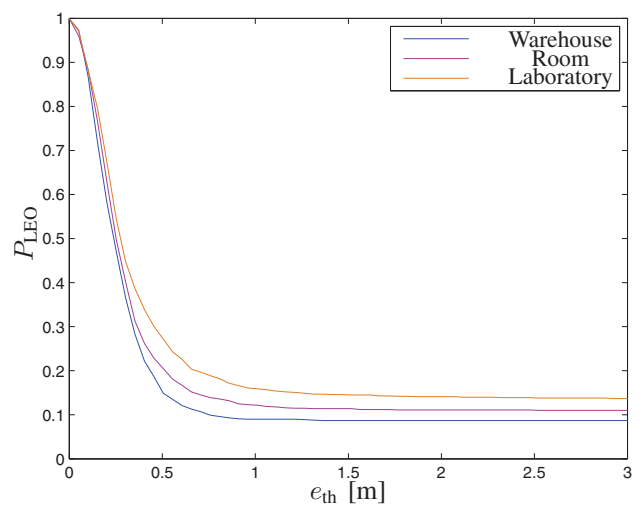

Fig. 7. LEO proabibilty over 1000 tag positions for three different scenarios.

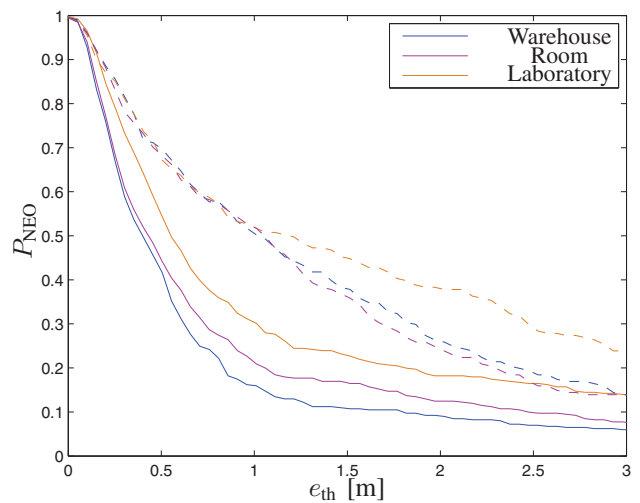

Fig. 8. NEO probability over 10 random trajectories each of length $20 \mathrm{~m}$ for $T_{\mathrm{r}}=1 \mathrm{~s}$ (solid) and $T_{\mathrm{r}}=0.5 \mathrm{~s}$ (dashed), and three different scenarios.

and LOS conditions [10]. The antenna-channel model derived from measurements is then used to evaluate TOA estimation accuracy in the presence of interfering tags. Specifically, we use the model to evaluate the localization and tracking performance with LS (static tags) and PF (dynamic tags) algorithms in the presence of 21 interferers located between $2 \mathrm{~m}$ and $8 \mathrm{~m}$ from each reader.

For static tags, Fig. 7 shows the LEO as a function of target localization error when LS algorithm is employed to estimate the location of 1000 positions in different environments. Note that, the localization error is below $0.37 \mathrm{~m}, 0.4 \mathrm{~m}$, and $0.56 \mathrm{~m}$ in the $75 \%$ of cases for the warehouse, room, and laboratory scenario, respectively.

For dynamic tags tracked via PF algorithm, a Gaussian distributed mobility model is considered, where at each instant the standard deviation depends on the uncertainty of the target movement, and the mean depends on the previous position estimate. The navigation algorithm estimates the tag position every $T_{\mathrm{r}}$ seconds and is based on the speed and direction learning (SDL) model, in which at each time $t_{k}=t_{0}+k T_{\mathrm{r}}\left(t_{0}\right.$ being the time of the first location estimate) the speed vector is determined from a window of previously estimated positions (five for the case study). We consider a Gaussian distributed perception model with standard deviation depending on both 
the TOA estimation technique and the propagation conditions. Details on the mobility and perception models can be found in [26]. Figure 8 shows the NEO as a function of target localization error over 10 random trajectories of a tag moving at $1 \mathrm{~m} / \mathrm{s}$ in different environments. In the worst case, that is the warehouse scenario, the navigation error is below $0.7 \mathrm{~m}$ and $2.07 \mathrm{~m}$ in the $75 \%$ of cases for $T_{\mathrm{r}}=0.5 \mathrm{~s}$ and $T_{\mathrm{r}}=1 \mathrm{~s}$, respectively.

\section{CONCLUSION}

In this paper we presented a novel UHF/UWB-RFID system with localization and tracking capabilities. The key technology of this system is the UWB backscattering modulation enabling localization features. In addition to UWB, an UHF signal is employed to ensure compatibility with RFID systems at $868 \mathrm{MHz}$, and to enable the tag wake-up for minimizing battery consumption and improve synchronization. The overall system together with the reader and tag architectures here presented have been developed in the scope of the SELECT European project. In particular, the reader includes two UWB chips, one for the transmitter and the other for the receiver, to realize a quasi-monostatic radar configuration. The tag architecture is based on three different chips connected to a UHF-UWB co-located antenna. The first one operates in the UHF band while the second one is connected to the UWB switch enabling passive backscattering schemes. Extensive measurement campaigns have been carried out to characterize a complete UWB backscattering antenna-channel model, which has been employed to evaluate the localization and tracking performance in different scenarios. Results take into account the hardware limitations, channel measured data, and interferences, showing that the system is able to provide submeter accuracy in localization and tracking for different environments. Further works will focus on the system integration and proof of concept in real environments.

\section{ACKNOWLEDGMENT}

This work has been performed within the framework FP7 European Project SELECT (grant no. 257544). Authors are thankful to Novelda A.S. and Iskra Sistemi.

\section{REFERENCES}

[1] J. Mentzer, W. DeWitt, J. Keebler, S. Min, N. Nix, C. Smith, and Z. Zacharia, "Defining supply chain management," Journal of Business logistics, vol. 22, no. 2, pp. 1-25, 2001.

[2] D. Dardari, R. D'Errico, C. Roblin, A. Sibille, and M. Win, "Ultrawide Bandwidth RFID: The Next Generation?" Proc. of the IEEE, vol. 98 , no. 9 , pp. $1570-1582,2010$.

[3] D. Dardari and R. D'Errico, "Passive Ultrawide Bandwidth RFID," in Global Telecommunications Conference, 2008. IEEE GLOBECOM 2008. IEEE, 302008.

[4] D. Arnitz, U. Muehlmann, and K. Witrisal, "UWB ranging in passive UHF RFID: proof of concept," Electronics Letters, vol. 46, no. 20, pp. $1401-1402,302010$.

[5] "Smart and Efficent Location, identification and Cooperation Techniques (SELECT)," http://www.selectwireless.eu/.

[6] D. Dardari, A. Conti, U. J. Ferner, A. Giorgetti, and M. Z. Win, "Ranging with ultrawide bandwidth signals in multipath environments," Proc. IEEE, vol. 97, no. 2, pp. 404-426, Feb. 2009, special issue on UltraWide Bandwidth (UWB) Technology \& Emerging Applications.
[7] Y. Shen and M. Z. Win, "Fundamental limits of wideband localization - Part I: A general framework," IEEE Trans. Inf. Theory, vol. 56, no. 10, pp. 4956-4980, Oct. 2010. [Online]. Available: http://arxiv.org/abs/1006.0888v1

[8] Y. Shen, H. Wymeersch, and M. Z. Win, "Fundamental limits of wideband localization - Part II: Cooperative networks," IEEE Trans. Inf. Theory, vol. 56, no. 10, pp. 4981-5000, Oct. 2010. [Online]. Available: http://arxiv.org/abs/1006.0890v1

[9] S. Gezici, Z. Tian, G. B. Giannakis, H. Kobayashi, A. F. Molisch, H. V. Poor, and Z. Sahinoglu, "Localization via ultra-wideband radios: a look at positioning aspects for future sensor networks," IEEE Signal Process. Mag., vol. 22, no. 4, pp. 70-84, Jul. 2005.

[10] R. D'Errico, “An indoor backscattering channel characterization for UWB passive RFID applications," in Antennas and Propagation (EUCAP), 2012 6th European Conference on, march 2012, pp. 1169 -1173.

[11] A. Sibille, M. Sacko, Z. Mhanna, F. Guidi, and C. Roblin, "Joint antenna-channel statistical modelling of uwb backscattering rfid," in Ultra-Wideband (ICUWB), 2011 IEEE International Conference on, sept. 2011, pp. $474-478$.

[12] A. Molisch, D. Cassioli, C.-C. Chong, S. Emami, A. Fort, B. Kannan, J. Karedal, J. Kunisch, H. Schantz, K. Siwiak, and M. Win, "A comprehensive standardized model for ultrawideband propagation channels," IEEE Transactions on Antennas and Propagation, vol. 54, no. 11, pp. $3151-3166$, nov. 2006

[13] D. Dardari, F. Guidi, C. Roblin, and A. Sibille, "Ultra-wide bandwidth backscatter modulation: Processing schemes and performance," EURASIP Journal on Wireless Communications and Networking, vol. 2011:47, no. 1, 2011

[14] F. Guidi, N. Decarli, D. Dardari, C. Roblin, and A. Sibille, "Performance of UWB backscatter modulation in multi-tag RFID scenario using experimental data," in IEEE International Conference on Ultra-Wideband, ICUWB 2011, Bologna, Italy, sep 2011, pp. 1-5.

[15] N. Decarli, F. Guidi, A. Conti, and D. Dardari, "Interference and clock drift effects in UWB RFID systems using backscatter modulation," in IEEE International Conference on Ultra-Wideband, ICUWB 2012, Syracuse, USA, sep 2012, pp. 1-5.

[16] D. Dardari, N. Decarli, A. Guerra, and A. Conti, "Enhanced localization coverage with Non- Regenerative UWB relay," in 20th European Signal Processing Conference 2012 (EUSIPCO 2012), Bucharest, Romania, Aug. 2012, pp. 1-5.

[17] S. Couronne, N. Hadaschik, M. Fassbinder, T. von der Grun, M. Weyn, M. Klepal, Widyawan, and T. Denis, "Locon - a platform for an interworking of embedded localisation and communication systems," in IEEE Conference on Sensor, Mesh and Ad Hoc Communications and Networks Workshops, SECON 2009, june 2009, pp. $1-3$.

[18] D. Lachartre, B. Denis, D. Morche, L. Ouvry, M. Pezzin, B. Piaget J. Prouve, and P. Vincent, "A 1.1nJ/b 802.15.4a-Compliant Fully Integrated UWB Transceiver in 0.13 $\mu \mathrm{m}$ CMOS," in ISSCC 2009, Proc. of the, San Francisco, Feb. 2009.

[19] G. Masson, D. Morche, H. Jacquinot, Vincent, Dehmas, S. Paquelet, A. Bisiaux, O. Fourquin, J. Gaubert, and S. Bourdel, “A 1 nJ/b 3.2-to-4.7 GHz UWB 50 Mpulses/s double quadrature receiver for communication and localization," in ESSCIRC, 2010 Proc. of the, 2010, pp. $502-505$.

[20] FCC, "Revision of Part 15 of the Commissions Rules Regarding UltraWideband Transmission Systems, First Report and Order, ET Docket 98-153, FCC 02-8, adopted/released Feb. 14/Apr. 22, 2002 ."

[21] "ECC/DEC/(06)12, amended 31 october 2008."

[22] M. Z. Win, A. Conti, S. Mazuelas, Y. Shen, W. M. Gifford, D. Dardari, and M. Chiani, "Network localization and navigation via cooperation," IEEE Commun. Mag., vol. 49, no. 5, pp. 56-62, May 2011.

[23] S. Bartoletti and A. Conti, "Passive network localization via UWB wireless sensor radars: the impact of TOA estimation," Bologna, Italy, Sep. 2011, pp. 576-580.

[24] A. Conti, M. Guerra, D. Dardari, N. Decarli, and M. Z. Win, "Network experimentation for cooperative localization," IEEE J. Sel. Areas Commun., vol. 30, no. 2, pp. 467-475, Feb. 2012.

[25] V. Heiries, K. Belmkaddem, F. Dehmas, B. Denis, L. Ouvry, and R. D'Errico, "UWB backscattering system for passive RFID tag ranging and tracking," in Ultra-Wideband (ICUWB), 2011 IEEE International Conference on, sept. 2011, pp. $489-493$.

[26] S. Bartoletti, M. Guerra, and A. Conti, "UWB passive navigation in indoor environment," in 4th Int. Symp. on Appl. Sci. in Biomed. and Commun. Technol., Barcelona, Spain, Oct. 2011, pp. 1-5. 